\title{
Integrierte gewässerökologische Modellansätze zur Beurteilung von Gewässervernetzungsvarianten am Beispiel der Unteren Lobau
}

\author{
Daniel Trauner · Andrea Funk · Eva-Maria Pölz • Eva Feldbacher · Gabriele Weigelhofer · Walter Reckendorfer • \\ Thomas Hein
}

Online publiziert: 13. Juli 2016

(c) Der/die Autor(en) 2016 . Dieser Artikel ist eine Open-Access-Publikation.

Zusammenfassung Weltweit zählen Fließgewässersysteme und Auenlandschaften zu den gefährdetsten Ökosystemen. Flussregulierungs- und Verbauungsmaßnahmen haben diese Systeme von ihren natürlichen Wasserstandsschwankungen bzw. dem Wasseraustausch mit dem Fluss entkoppelt und führen damit $\mathrm{zu}$ einer Verlandung ehemaliger hochdynamischer Habitate. Gezielte Managementmaßnahmen können diesen Entwicklungen entgegenwirken, müssen vor Umsetzung aber auf ihre Auswirkungen hin überprüft werden. Grundsätzlich initiieren alle Restaurationsmaßnahmen grundlegende Prozesse im Fluss-Auensystem, auf die Arten mit unterschiedlichen Habitatansprüchen und unterschiedlichem Schutzstatus verschieden reagieren können. Eine Möglichkeit einer wissenschaftlich fundierten Prognose von hydrologischen Managementmaßnahmen wird in diesem Artikel am Beispiel der Verwendung von Habitatmodellen für eine Studie in der Unteren Lobau vorgestellt. Die Untere Lobau ist eine Auenlandschaft im Osten von Wien, nördlich der Donau. Sie

MSc. D. Trauner $(\bowtie)$.

Mag. Dr. A. Funk .

Mag. E.-M. Pölz · DI E. Feldbacher .

Mag. Dr. G. Weigelhofer ·

Assoc- Prof. Dr. T. Hein

Department für

Wasser-Atmosphäre-Umwelt,

Institut für Hydrobiologie

und Gewässermanagement,

WasserCluster Lunz - Biologische

Station GmbH/Universität

für Bodenkultur Wien,

Max-Emanuel-Straße 17, 1180 Wien,

Österreich

E-Mail: daniel.trauner@boku.ac.at

Dr. W. Reckendorfer

VERBUND Hydro Power GmbH, Wien, Österreich ist Teil des Nationalparks Donau-Auen und geschützter FFH-Lebensraum. Vor der Donauregulierung Ende des 19. Jahrhunderts war die Untere Lobau ein dynamisches Auengebiet, aber die heute vorherrschenden Verlandungsprozesse bedrohen dieses in Europa selten gewordene Ökosystem. Für dieses Gebiet wurden drei Managementvarianten untersucht: 1) Nullvariante Das Ausbleiben jeglicher Maßnahmen mit einer Fortsetzung der Verlandungsprozesse; 2) Dotation - Die Zufuhr einer gerade ausreichenden Menge an Wasser, um Wasserflächen gemäß dem Status quo zu erhalten; 3) Anbindung - Das Herstellen einer stromaufwärtigen Verbindung zum Hauptstrom der Donau, was eine Rheophilisierung des Systems und zumindest eine Annäherung an historische Verhältnisse darstellt. Auf Basis modellierter Umweltdaten und Habitatpräferenzen wurden mittels binär-logistischer Regressionen von ausgewählten Arten verschiedenster Gruppen potenzielle Habitatflächen (Weighted Usable Areas) berechnet. Die Qualität der Modellergebnisse konnte unterstreichen, dass die Modellierungen die hydrologischen Maßnahmen mit ihrem Einfluss auf die Biozönose gut beschreiben und damit eine wertvolle Hilfe in der Entscheidungsfindung im Gewässermanagement sein können.

Schlüsselwörter Auen .

Restaurationsmaßnahmen .

Habitatmodelle .

Managementoptionen · Nationalpark

Integrated hydro-ecological modelling approaches to assess reconnection options of water bodies - A case study of the Lower Lobau

Abstract Floodplains and wetlands are among the most endangered ecosystems worldwide. River-regulation and flood protection measures cut off these formerly highly dynamic systems from their natural water level fluctuations and water exchange conditions leading to siltation processes. Restoration measures to counteract these developments need to be assessed for the effect on currently established habitats and communities in order to estimate their effects, as species with different habitat preferences and different protection status do react distinctly different. This article will present how habitat modelling was used in a case study of the Untere Lobau to assess and predict the effects of potential management measures. The Untere Lobau is a wetland ecosystem of the Danube east of Vienna. It is part of the national park Donau-Auen and a protected area according to the EU habitat-directive. Prior to the river regulation, at the end of the $19^{\text {th }}$ century, the Untere Lobau was a dynamic floodplain. Today, siltation processes endanger especially the status of the aquatic habitats. Three management options were investigated: 1) business as usual - No implementation of additional hydrological measures, thus siltation processes are not mitigated; 2 ) a water enhancement scheme - A small amount of water is supplied to preserve the water bodies at the current status quo; 3) partly reconnection - An upstream reconnection of the floodplain to the main channel of the Danube, leading to a more rheophilic characteristic of the system moving towards conditions prior regulation. Based on a model approach and calculating habitat preferences via binary logistic regressions of selected species from different organism groups, an increase or decrease of available suitable habitat area (weighted usable areas) could be estimated. This study proofed clearly that models can assess the effects of hydrological management measures on the 
biocenosis and that they are a valuable tool for supporting the decision taking process in wetland management.

\section{Einleitung}

Fließgewässersysteme und Auenlandschaften bieten einen diversen Lebensraum für eine Vielzahl an aquatischen und semiaquatischen Tier- und Pflanzenarten. Gleichzeitig zählen diese Habitate $\mathrm{zu}$ den weltweit gefährdetsten Ökosystemen (Brinson und Malvarez 2002; Tockner und Stanford 2002; Malmqvist und Rundle 2002). Entlang der gesamten Donau beträgt der Verlust an ursprünglichen Auengebieten $68 \%$ (e. g. Sommerwerk et al. 2010). An der oberen Donau sind sogar $90 \%$ der ursprünglichen Überschwemmungsgebiete vom Hauptstrom der Donau abgetrennt und nur mehr $15 \%$ der Strecke vom Ursprung bis zur österreichisch-slowakischen Grenze gelten als freie Fließstrecken (Sommerwerk et al. 2009). Umso wichtiger sind der Erhalt und die Wiederanbindung abgetrennter Auenlandschaften an den Hauptstrom der Donau. Das Restaurationspotenzial entlang der Donau wurde bereits in mehreren Studien untersucht (Schwarz 2010; Hein et al. 2015). Der Abschnitt Wien - Bratislava, der vor der Donauregulierung im 19. Jahrhundert durch eine hochdynamische Auenlandschaft mit einer Ausdehnung von $303 \mathrm{~km}^{2}$ charakterisiert war, umfasst nunmehr einzelne Auenabschnitte mit unterschiedlichem Restaurationspotenzial. $98 \mathrm{~km}^{2}$ der noch vorhandenen Auenlandschaft wurden seit 1996 zum Großteil durch die Errichtung des Nationalpark Donau-Auen geschützt und bereits teilweise restauriert (Schiemer et al. 2007; Reckendorfer et al. 2005). Eine weitere Fläche im Ausmaß von $60 \mathrm{~km}^{2}$, welche ebenfalls teilweise im Nationalparkgebiet liegt, weist noch Restaurationspotenzial auf (Hein et al. 2015). Das Nebeneinander von feuchten und trockenen Standorten bedingt den großen Artenreichtum von Auenlandschaften, so auch der Unteren Lobau - Teil des Nationalparks DonauAuen sowie Natura-2000-Gebiet. Viele Arten der Roten Liste und nach europäischen Richtlinien geschützte Arten haben hier ihren Lebensraum. Jedoch ist der langfristige Erhalt des Auencharakters massiv gefährdet (Hein et al. 2006). Seit der Donauregulierung Ende des 19. Jahrhunderts ist die Untere Lobau vom Hauptstrom entkoppelt und die morphologische Dynamik stark zurückgegangen (Hohensinner et al. 2013). Der Geschieberückhalt der Kraftwerkskette stromaufwärts des Gebiets bedingt die Eintiefung der Donau. All dies führte im Lauf der Jahrzehnte zum Absinken des Grundwasserniveaus, zu einer Verminderung der auentypischen Dynamik und der damit verbundenen Umlagerungen, zu Verlandungsprozessen der Augewässer und in der Folge zu ökologischen Defiziten (Reckendorfer et al. 2005) und einer Verschiebung der Artenzusammensetzung.

Um die grundlegenden Funktionen der Unteren Lobau als Lebensraum für zahlreiche gefährdete Arten (FFHRichtlinien, Vogelschutzrichtlinie etc.) zu erhalten, sind daher Restaurierungsbzw. Management-Maßnahmen unumgänglich. Dazu können grundsätzlich zwei Ansätze gewählt werden: i) Ein stark konservierender Ansatz, der zum Ziel hat, den derzeitigen Zustand der Lobau zu erhalten, indem die hydrologischen Verhältnisse verbessert werden und den Verlandungstendenzen entgegengewirkt wird. Das könnte durch eine Dotation in Form einer geregelten Einspeisung von geringen Wassermengen (wenige $\mathrm{m}^{3} / \mathrm{s}$ ) mit kontrollierter Qualität erreicht werden, wie sie bereits in der Oberen Lobau realisiert wurde (Weigelhofer et al. 2013). ii) Ebenso kann ein restaurierender Ansatz gewählt werden, der zum Ziel hat, die ursprünglichen dynamischen Verhältnisse durch eine verstärkte Wiederanbindung an die Donau, soweit es die Rahmenbedingungen im Gebiet erlauben, wiederherzustellen. Eine derartige Wiederanbindung wurde z. B. in Regelsbrunn, den Orther Auen oder auch im Bereich des Mannsdorfer Hagels durchgeführt (Schiemer et al. 1999; Reckendorfer et al. 2005, 2013).

Augewässer sind komplexe Systeme, in denen verschiedene hydrologische Gradienten wirksam sein können, zum Beispiel der Gradient von dynamischen, permanent durchströmten Seitenarmen zu lentischen, isolierten Gewässern oder von Armen mit permanenter Wasserführung zu temporären, semiaquatischen Bereichen. Die Prognose der Wirkungen verschiedener Maßnahmen auf die Artengemeinschaft braucht demnach gezielte Modellansätze, die robuste Aussagen liefern können. Dazu wird in dieser Arbeit eine integrative Bewertungsmethode auf Basis einer quantitativen Habitatmodellierung vorgestellt und für das Gebiet der Unteren Lobau als Fallstudie getestet. Die Modellierung berücksichtigt vor allem naturschutzfachlich relevante Tiere und Pflanzen (Lebensräume), welche den gesamten Gradienten von Augewässern abdecken, sowie morphologische und physikalische Parameter, die die unterschiedlichen Lebensräume charakterisieren.

\section{Methoden}

\subsection{Gebiet}

Das Gebiet der Unteren Lobau liegt östlich von Wien und linksufrig der Donau von Strom-Kilometer 1918 bis 1908 . Sie umfasst eine Fläche von 1474 ha und besteht aus $61 \%$ Auwald, $13 \%$ trockenen Schotterflächen, $12 \%$ permanenten und intermittierenden $\mathrm{Ge}$ wässern sowie Schilf, 7 \% Wiesen und $7 \%$ Feldern. Bei einem theoretischen maximalen Durchfluss der Donau von $14000 \mathrm{~m}^{3} / \mathrm{s}$ hat die Untere Lobau eine Retentionskapazität von 47,6 Mio. $\mathrm{m}^{3}$ (Funk et al. 2013). Seit den Flussregulierungen im 19. Jahrhundert haben dynamische Prozesse in der Unteren Lobau stetig abgenommen (Hohensinner et al. 2013). Da die Auen-Biotope der Unteren Lobau in Mitteleuropa Seltenheitswert haben, wurde sie 1977 zum UNESCOBiosphärengebiet ernannt und ist seit 1982 Ramsar-Gebiet. Im Jahr 1996 wurde sie Teil des Nationalparks DonauAuen, und im Jahr 2000 erklärte man die Untere Lobau zum Natura-2000-Gebiet. Die Gewässer der Unteren Lobau bestehen aus einem ehemals durchflossenen Nebenarm der Donau (welcher heute stromabwärts oberflächig an den Hauptarm der Donau angebunden ist) und einer Reihe von abgetrennten Nebenarmen und isolierten Wasserkörpern (Abb. 1). Gegenwärtig ist das System grundwassergespeist und wird von stromabwärtiger Seite her geflutet (Funk et al. 2013, 2009; Weigelhofer et al. 2011; Baart et al. 2010).

\subsection{Varianten der Managementmaßnahmen}

Die grundlegende Idee der Prognosemodelle war $\mathrm{zu}$ untersuchen, welche Folgen sich bei unterschiedlichen hydrologischen Maßnahmen für die ausgewählten Tiere, Pflanzen und Lebensräume in der Unteren Lobau ergeben würden. Hierfür wurden drei hypothetische Management-Varianten formuliert: 


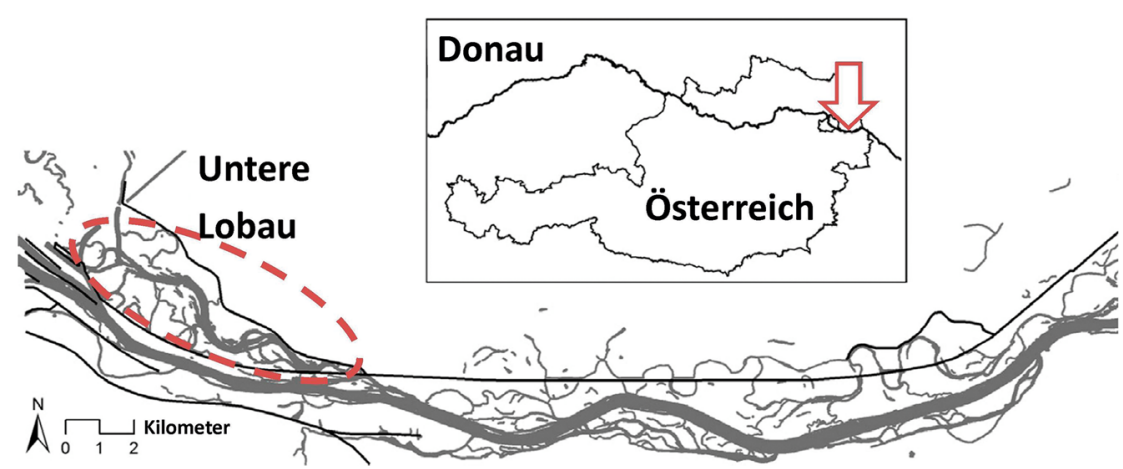

Abb. 1 Untersuchungsgebiet Untere Lobau; grau-Wasserflächen, schwarz-Hochwasserschutzdamm (abgeändert übernommen von Funk et al. 2013)

Die Nullvariante beschreibt ein $\mathrm{Zu}$ kunftsszenario, bei dem keinerlei Maßnahmen gesetzt werden und die Verlandung der Gewässer somit unbeeinträchtigt fortschreiten würde.

Zum Erhalt des Status quo (konservierende Maßnahme) wurde bei dieser Variante von einer Dotation mit geringen Wasserzugaben aus der Neuen Donau ausgegangen. Diese Variante sieht eine relativ konstante Einleitung von wenigen Kubikmetern pro Sekunde im oberen Bereich des Gebiets vor, um den Wasserhaushalt der Unteren Lobau zu verbessern. Das erhöhte Wasserangebot soll zu einem Stopp der derzeitigen Austrocknung des Gebiets führen und so zumindest den derzeitigen Zustand sichern. Diese Variante orientiert sich an der Arbeit von Baart et al. (2010), in der gezeigt wurde, dass schon eine geringe Dotation den Wasserhaushalt verbessert.

Für die dritte Variante, eine eingeschränkte Dynamisierung und Rheophilisierung der Unteren Lobau, wurde eine teilweise Anbindung des Systems an die Donau mit variierenden Wassermengen von bis zu $80 \mathrm{~m}^{3} / \mathrm{s}$ je nach Wasserstand der Donau angenommen. Diese Variante stellt eine Annäherung an die historischen Verhältnisse vor der Regulierung der Donau dar (Hohensinner et al. 2013), wobei jedoch die ursprüngliche Dynamik bei weitem nicht erreicht wird. Bei dieser Maßnahme müsste sich nicht nur eine Verbesserung des Wasserangebots durch erhöhte Oberflächen- und Grundwasserstände, welche zusätzliche Flachgewässer und neue temporäre Gewässer entstehen lassen, sowie eine Erhöhung der Anbindungsdauer in den Gewässern der Unteren Lobau einstellen, sondern es sollten auch durchgängig strömende Verhältnisse im Hauptarm ähnlich wie schon bei anderen Wiederanbindungen an die Donau erreicht werden (Hein et al. 1999; Schiemer et al. 1999; Tockner et al. 1999; Baart et al. 2010).

\subsection{Habitatmodellierung und Bewertung}

Die Prognose der Auswirkungen der drei Varianten stützte sich auf eine Habitatmodellierung (siehe Abb. 2). Bei der Wahl der Indikatorarten wurde vor allem darauf geachtet, dass der gesamte hydrologische Gradient der Augewässer abgedeckt ist. Es wurden sowohl Arten und Lebensraumtypen einbezogen, die in der Lobau im derzeitigen Zustand eine starke Verbreitung haben, als auch Arten und Lebensraumtypen, die in restaurierten, dynamischen Gewässersystemen des Nationalparks verbreitet sind. Dazu wurden Arten aus den Gruppen der Amphibien, Fische, Reptilien und Vögel sowie Lebensraumtypen nach der Fauna-Flora-Habitat-Richtlinie (FFH-Richtlinie 1992) nach folgenden Kriterien ausgewählt: Schutzstatus (geschützte bzw. gefährdete Arten wurden bevorzugt), Vorkommenshäufigkeit in der Unteren Lobau und im restlichen Nationalpark, Modellierbarkeit (ausreichende Daten) und Autökologie (Habitatansprüche). Biologische Daten wurden im Rahmen des Projekts erhoben bzw. konnte auf vorhandene Daten aus dem Nationalpark Donau-Auen aus vorhergehenden Restaurierungsprojekten zurückgegriffen werden (Funk et al. 2013). Zu den modellierten Arten zählten beispielsweise der Wels (Silurus glanis, Linnaeus 1761), die Nase (Chondrostoma nasus, Linnaeus 1761), die Rotbauchunke (Bombina bombina, Linnaeus 1761), der Donau-Kammmolch (Triturus dobrogicus, Kiritzescu 1903) und die Libellenart Große Moosjungfer
(Leucorrhinia pectoralis, Charpentier 1825). Unter den modellierten FFHLebensraumtypen waren unter anderem oligo- bis mesotrophe kalkhaltige Gewässer mit benthischer Vegetation aus Armleuchteralgen, Weichholzau (FFH LRT 91E0), Hartholzau (FFH LRT 91F0), oder auch naturnahe Kalk-Trockenrasen (FFH LRT 6210) vertreten.

Die hydrologischen Grundlagen, um Aussagen zur Entwicklung von Arten und Habitaten treffen zu können, wurden von der Gruppe Wasser ${ }^{\circledR}$, der DonauConsult Ingenieurbüro $\mathrm{GmbH}$ und der Technischen Universität Wien, Institut für Wasserbau und Ingenieurbiologie, zur Verfügung gestellt. Folgende abiotische Parameter wurden für die Berechnung der Habitatmodelle gewählt (Funk et al. 2013):

- Wasserflächen sind wichtig, um Verluste von Gewässern durch Verlandung und Auflandung bzw. einen Zuwachs an Gewässerflächen durch einen maßnahmenbedingten Anstieg der Wasserspiegel berücksichtigen zu können.

- Die maximalen Wassertiefen in den Augewässern für Regulierungsniederwasser (RNW), Mittelwasser (MW) und den höchsten Schifffahrtswasserstand (HSW) in der Donau sowie Wasserstandsschwankungen zwischen RNW und HSW für die unterschiedlichen Gewässerabschnitte bilden die Permanenz bzw. Stabilität von Gewässern ab und sind beispielsweise bedeutend für die Diversität von Amphibien (Kolozsvary und Swihart 1999).

- Die Anbindungsdauerund die Durchströmungsdauer beschreiben den Gradienten von permanent strömenden/angebundenen $\mathrm{zu}$ stehenden Verhältnissen und sind ebenso wichtige Surrogatparameter für verschiedene physikalische Parameter wie Substratbeschaffenheit und Wasserqualität und dementsprechend bedeutsam für die Biozönose. Die Anbindungsdauer beschreibt die Häufigkeit von Überschreitungen jener Wasserstände, ab welchen die verschiedenen Wasserkörper im Untersuchungsgebiet zumindest einseitig an die Donau angebunden sind. Der Parameter Durchströmung erfasst die Anzahl der Tage im Jahr, an denen ein Wasserkörper durchgehend strömende Verhältnisse aufweist.

- Die Fließgeschwindigkeit ist bedeutend für strömungsliebende bzw. strömungsmeidende Organismen. 


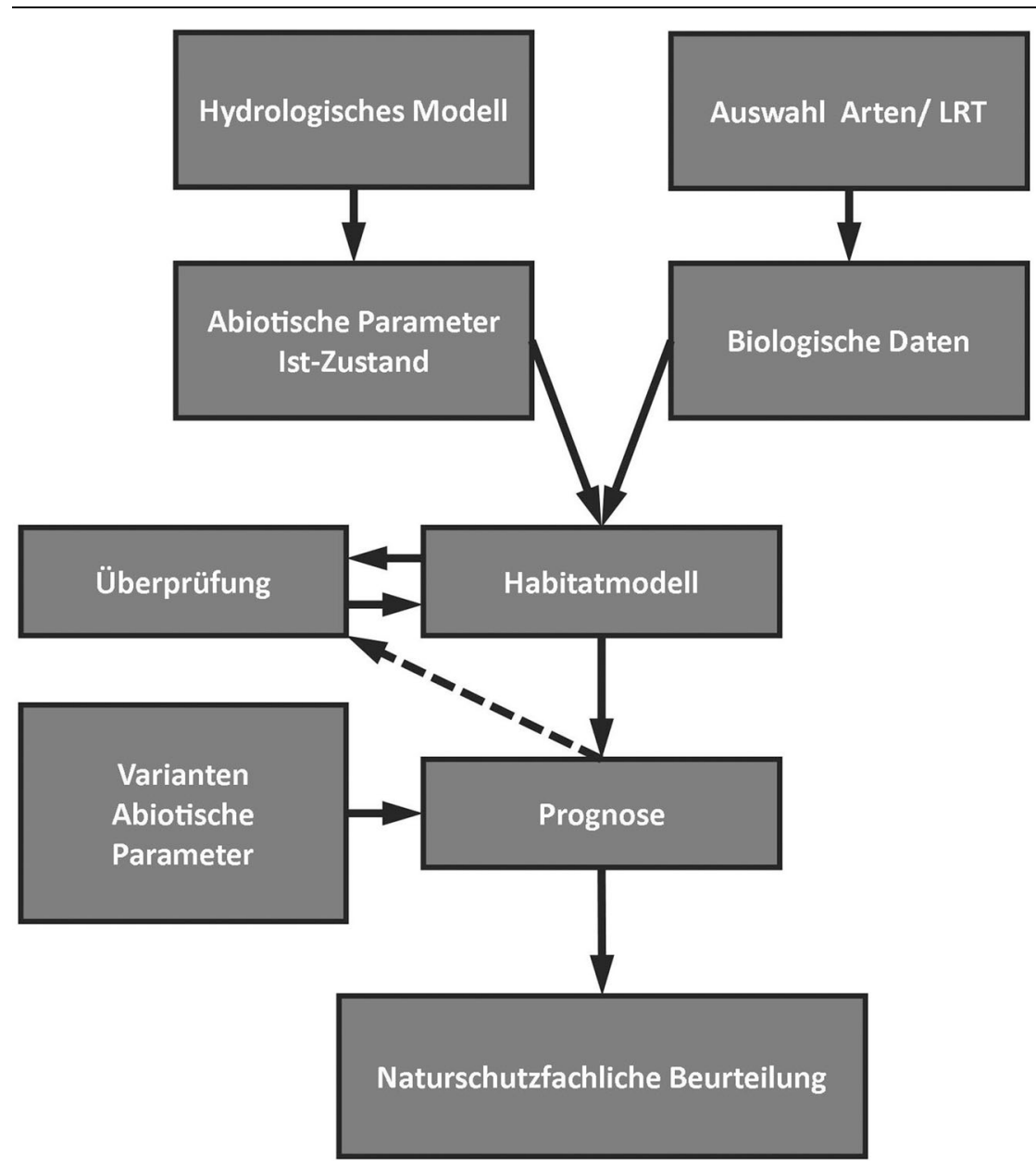

Abb. 2 Schema zur Habitatmodellierung, und Prognose der Maßnahmenvarianten

- Die Sonneneinstrahlung repräsentiert die Produktivität und das Klima der Wasserkörper und ist beispielsweise bedeutend für die Biodiversität von Makrophyten (Baart et al. 2010). Berechnet wurde die Globalstrahlung, also die Summe aus der direkten und der diffusen Sonneneinstrahlung auf einer Fläche, mittels Solar Radiation Tool in ARCGIS 10.2. Als Grundlage wurde ein digitales Höhenmodell verwendet.

Für die Habitatmodellierungen wurden binär-logistische Regressionen angewendet, eine eine in diesem Zusammenhang häufig verwendete Methode. Die Methodik ist in Funk et al. (2013) im Detail beschrieben. Mit binär-logistischen Regressionen wurde die An- oder Abwesenheit einer Art unter bestimmten abiotischen Rahmenbedingungen modelliert. Die Methode zeigt eine gute Vorhersagequalität im Vergleich $\mathrm{zu}$ anderen Ansätzen und wird häufig verwendet, um die Habitatverfügbarkeit für Arten zu prognostizieren (Ennis et al. 1998; Elith und Graham 2009; Funk et al. 2013). Mittels Rückwärtsselektion wurden die nicht signifikanten Variablen aus dem Regressionsmodell ausgeschlossen, die anhand des bestehenden Datensatzes überprüft werden konnten. Das Ergebnis der Modelle war die aus den abiotischen Parametern berechnete Auftretenswahrscheinlichkeit einer Art. Sie liegt zwischen 0 „sicher nicht vorhanden“ und 1 „mit Sicherheit anzutreffen".

Überprüft wurden die Modelle mittels „bootstrapping“ $(0.632$ + bootstrapcrossvalidation technique, Gerds et al. 2008; Wintle et al. 2005; Efron und Tibshirani 1997). Diese Methode wird als die sicherste Überprüfung der Prognosequalität von Modellen gesehen, wenn kein unabhängiger Datensatz für die Überprüfung zur Verfügung steht. Dazu wird mehrmals eine Zufallsauswahl aus den Modelldaten entnommen und mittels „Area under the Receiver Operating Characteristic curve" (ROC curve; area under curve - AUC, Hanley und Mc-
Neil 1982) überprüft, ob die Einstufung der einzelnen Modellflächen bezüglich ihrer Habitatqualität zufriedenstellend ist. Ein AUC-Wert unter 0,5 hat keine Prognosequalität, ein Wert von 1 bedeutet eine perfekte Prognose. Werte unter 0,7 werden als schwache Vorhersagequalität und Werte über 0,7 als gute Vorhersagequalität gewertet.

In einem zweiten Schritt wurden die Habitatmodelle mit den hydrologischen Prognosemodellen $\mathrm{zu}$ ökologischen Prognosemodellen für die einzelnen modellierten Arten und Lebensraumtypen verknüpft. Für den Vergleich der Varianten wurde dann aus den ökologischen Prognosemodellen die Wahrscheinlichkeit der Habitatverfügbarkeit für jede Art und jeden Lebensraumtyp mittels „Weighted usable areas“ (WUA, gewichtete nutzbare Fläche; Bovee 1986) berechnet.

Die WUAs geben die für die jeweilige Art oder den Lebensraumtyp potenziell nutzbare Fläche bei einer bestimmten Variante wieder. Die WUAs wurden nach folgender Formel berechnet:

$$
W U A=\sum_{i=1}^{n} \operatorname{prob}_{i} A_{i}
$$

Wobei probi die Wahrscheinlichkeit des Vorkommens einer/s bestimmten Art/Lebensraumtyps in einem Areal i (berechnet aus den binär logistischen Regressionsmodellen) und $\mathrm{A}_{\mathrm{i}}$ die Fläche dieses Areals ist (Funk et al. 2013).

Abschließend wurden alle drei Varianten für die modellierten Organismen und Lebensraumtypen mit dem Ist-Zustand verglichen und ihr Potenzial für eine Verbesserung bzw. Verschlechterung gegenüber der jetzigen Situation eingeschätzt.

\section{Ergebnisse und Diskussion}

\subsection{Habitatmodelle und biologische Prognosen}

Die Prognosemodelle waren für alle Arten und Lebensraumtypen von guter Qualität und hochsignifikant. In Abb. 3 sind die Ergebnisse der Habitatpräferenzen (binär-logistische Regressionen) ausgewählter Arten und Lebensraumtypen dargestellt. Die gewählten Parameter, Arten und Lebensraumtypen deckten den Gradienten gut ab und eigneten sich dafür, die gewählten Varianten genau zu beschreiben. Die Prognosemodelle der WUAs für ausgewählte Arten und Lebensraumtypen 

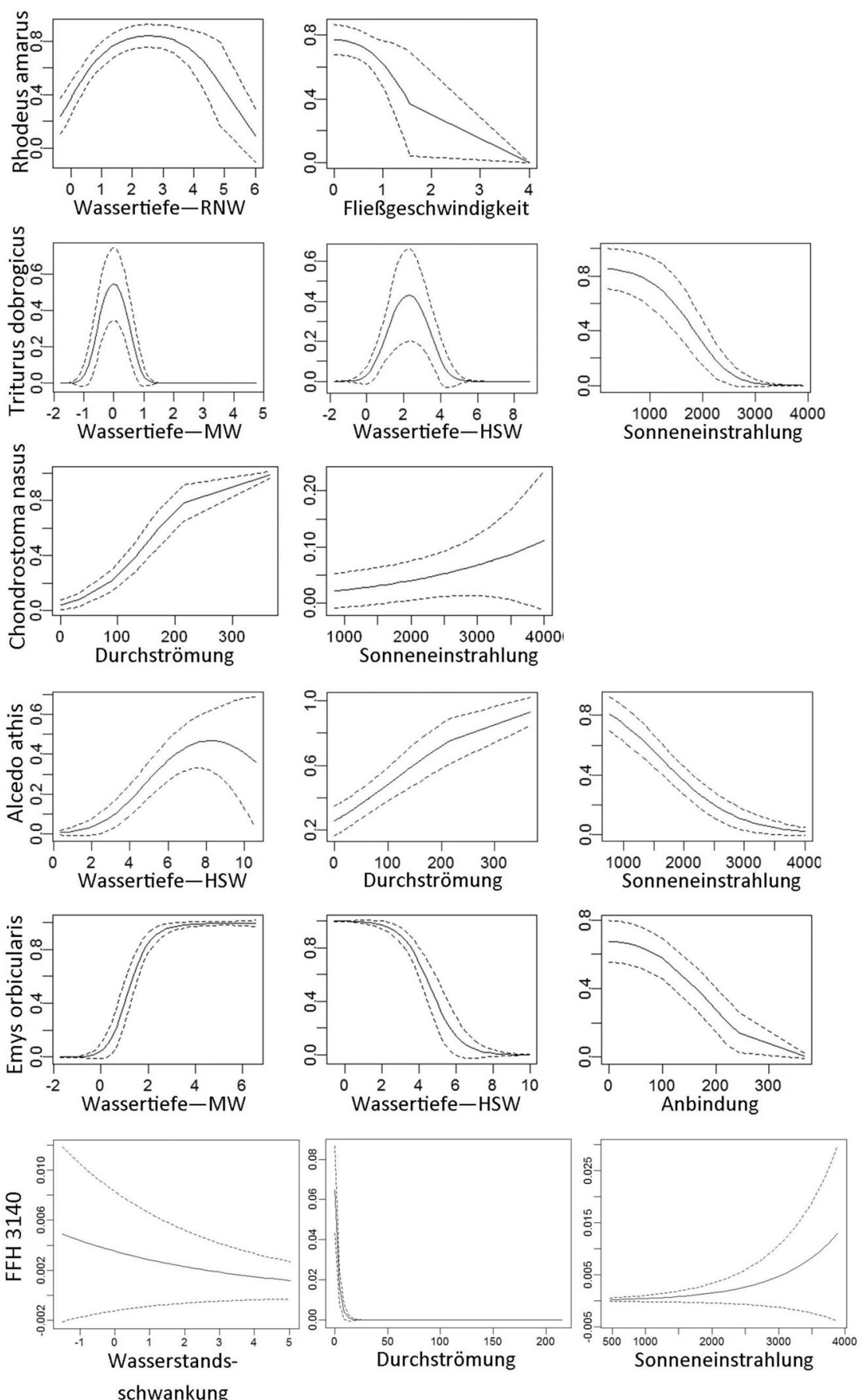

Abb. 3 Vorkommenswahrscheinlichkeiten (binär-logistische Regressionen; Median + Standardfehler) von ausgewählten modellierten Arten pro signifikanter Variable. RNW Regulierungsniederwasser, MW Mittelwasser, HSW Höchster Schifffahrtswasserstand. Rhodeus amarus (Bloch 1782) - Bitterling, Triturus dobrogicus (Kiritzescu 1903)-Donau-Kammmolch, Chondrostoma nasus (Linnaeus 1758)-Nase, Alcedo atthis (Linnaeus 1758) - Eisvogel, Emys orbicularis (Linnaeus 1758) -Europäische Sumpfschildkröte, FFH 3140 - oligo- bis mesotrophe kalkhaltige Gewässer mit benthischer Vegetation aus Armleuchteralgen sind in Abb. 4 dargestellt. Beispielsweise zeigte sich für den Bitterling (Rhodeus amarus, Bloch 1782), der geringe bis mittlere Strömungsgeschwindigkeiten bevorzugt und bis auf temporäre $\mathrm{Ge}$ wässer weit verbreitet ist (Guti 1996; Schiemer und Waidbacher 1992; Schiemer und Spindler 1989), sowie den Donau-Kammmolch (Triturus dobrogicus, Kiritzescu 1903), welcher flachere, dynamische Bereiche mit Wasserstandsschwankungen und geringer bis mittlerer Sonneneinstrahlung bevorzugt (Oldham et al. 2000; Maletzky et al. 2007), ein relativ geringer Einfluss durch die Änderung der physikalischen Bedingungen in den drei Varianten. Bei diesen beiden Arten ist nahezu nur der Zuwachs beziehungsweise Verlust von Gewässerflächen relevant. Andere Arten reagierten eindeutig positiv auf eine Rheophilisierung des Systems. Die Nase (Chondrostoma nasus, Linnaeus 1758), die breite und durchströmte Gewässer bevorzugt (Guti 1996; Schiemer und Waidbacher 1992; Schiemer und Spindler 1989) sowie der Eisvogel (Alcedo atthis, Linnaeus 1758), welcher tiefe und schmale durchströmte Gewässer mit Uferabriss als Habitat benötigt (Frühauf et al. 2006), sind prognostizierte Gewinner einer erhöhten Anbindung. Für die Dotation zeigt sich, dass die europäische Sumpfschildkröte (Emys orbicularis, Linnaeus 1758) mit Habitatpräferenzen für permanente Gewässer mit geringer Anbindung (Ficetola et al. 2004) und der FFH-Lebensraum 3140, also oligo- bis mesotrophe kalkhaltige Gewässer mit benthischer Vegetation aus Armleuchteralgen, wiederum diese Variante präferieren. Die Nullvariante zeigte durchgehend eine Verschlechterung der Bedingungen für die gewählten Organismen.

Die ökologischen Prognosemodelle zeigten die Auswirkungen einer Änderung der Abiotik auf die voraussichtliche Entwicklung der in der Unteren Lobau vorkommenden Organismen und Lebensräume anschaulich. Was allerdings nur indirekt berücksichtigt wurde, sind die Interaktionen zwischen den einzelnen Organismen, wie z. B. der Wettbewerb um denselben Raum bzw. dieselbe Nahrungsressource oder Räuber-Beute-Beziehungen. Eine stärkere Einbeziehung dieser Interaktionen würde die Aussagekraft der Modelle weiter verstärken, allerdings auch deren Komplexität erhöhen. Insgesamt konnte die Studie jedoch zeigen, dass sich der gewählte Modellansatz ideal eignet, 

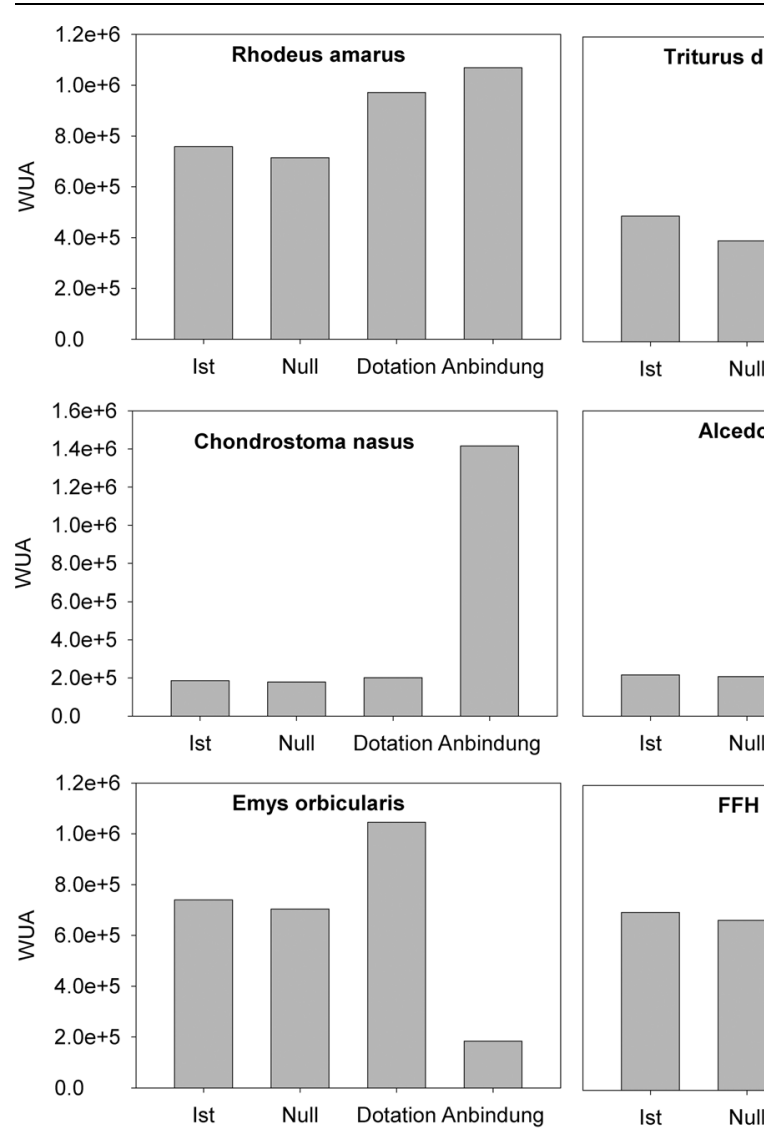

Ist

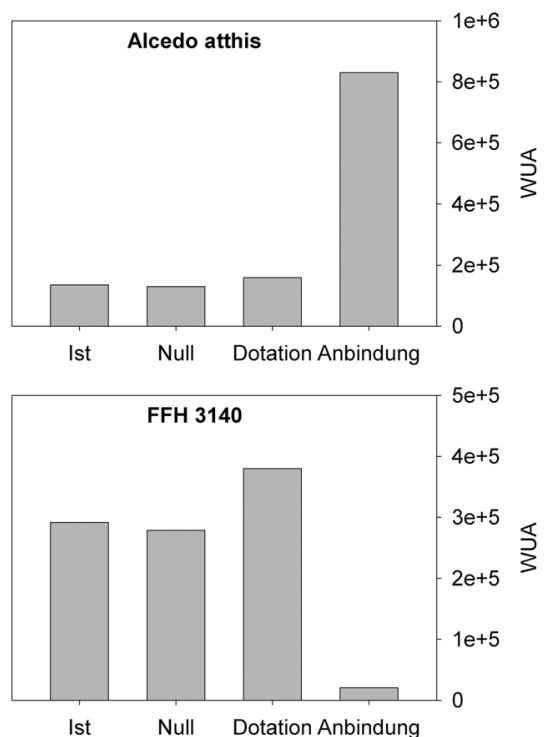

Abb. 4 Beispiel für die Ergebnisse der Prognosemodelle (Habitatmodellierung) dargestellt als „weighted usable area“ (WUA) für ausgewählte Arten und Lebensraumtypen. Rhodeus amarus (Bloch 1782) - Bitterling, Triturus dobrogicus (Kiritzescu 1903)Donau-Kammmolch, Chondrostoma nasus (Linnaeus 1758) - Nase, Alcedo atthis (Linnaeus 1758) - Eisvogel, Emys orbicularis (Linnaeus 1758) - Europäische Sumpfschildkröte, FFH 3140 Habitat-oligo- bis mesotrophe kalkhaltige Gewässer mit benthischer Vegetation aus Armleuchteralgen

um widersprüchliche Gebietsansprüche von Organismen beziehungsweise unterschiedliche Reaktionen auf wasserbauliche Maßnahmen abzubilden und "Gewinner" oder „Verlierer“ bei den verschiedenen Varianten zu identifizieren. Dies erleichtert die Formu- lierung von Kompromissvarianten. Die semodellen auf der Basis hydrologischer Modelle stellt somit eine wichtige Unterstützung bei der Entscheidungsfindung im Gewässermanagement von Fluss-Augebieten dar. Entwicklung von ökologischen Progno-

\section{Danksagung}

Wir möchten diesen Artikel Dr. Werner Pekarek $(\dagger)$ widmen, der ganz wichtig für die Projektdurchführung war und leider viel $\mathrm{zu}$ früh von uns gegangen ist. Die Finanzierung des Projekts erfolgte durch die Magistratsabteilung 45 der Stadt Wien, die EU (Europäischer Landwirtschaftsfonds zur Entwicklung des ländlichen Raumes) und das Bundesministerium für Landund Forstwirtschaft, Umwelt und Wasserwirtschaft. Wir bedanken uns bei der Gruppe Wasser ${ }^{\circledR}$, der DonauConsult Ingenieurbüro $\mathrm{GmbH}$ und dem Institut für Wasserbau und Ingenieurbiologie der Technischen Universität Wien für die hydrologischen Grundlagen. Unser besonderer Dank gilt außerdem der Nationalpark Donau-Auen $\mathrm{GmbH}$, der Magistratsabteilung 22 und der Magistratsabteilung 49, Gerhard Nagel, Thomas Ofenböck, Paul Blaschke, Raimund Taschke, Hannes Gabriel, Stefan Preiner, Maria Schindler, Andrea Waringer-Löschenkohl, Christian Schulze, Michael Schabuss und Georg Wolfram.

Open access funding provided by University of Natural Resources and Life Sciences Vienna (BOKU).

Open Access Dieser Artikel wird unter der Creative Commons Namensnennung 4.0 International Lizenz (http:// creativecommons.org/licenses/by/4. 0/deed.de) veröffentlicht, welche die Nutzung, Vervielfältigung, Bearbeitung, Verbreitung und Wiedergabe in jeglichem Medium und Format erlaubt, sofern Sie den/die ursprünglichen $\mathrm{Au}$ tor(en) und die Quelle ordnungsgemäß nennen, einen Link zur Creative Commons Lizenz beifügen und angeben, ob Änderungen vorgenommen wurden.

\section{Literatur}

Baart, I., Gschöpf, C., Blaschke, A. P., Preiner, S., Hein, T. (2010): Prediction of potential macrophyte developmentin response to restoration measures in an urban riverine wetland. Aquat. Bot. 93, 153-162.

Bovee, K. D. (1986): Development and evaluation of habitat suitability criteria for use in the instream flow incremental methodology. Biological Report 86: 235 pp., US Fish and Wildlife Service.

Brinson, M. M., Malvárez, A. I. (2002): Temperate freshwater wetlands: types, status, and threats. Environmental conservation, 29(02), 115-133.
Efron, B., Tibshirani, R. (1997): Improvements on crossvalidation: the 632 + bootstrap method J. Am. Stat. Assoc. 92, 548-560.

Elith, J., Graham, C. H. (2009): Do they? How do they? Why do they differ? On finding reasons for differing performances of species distribution models. Ecography 32, 66-77.

Ennis, M., Hinton, G., Naylor, D., Revow, M.

Tibshirani, R. (1998): A comparison of statisti-

cal learning methods on the GUSTO database. Stat. Med. 17, 2501-2508.

FFH-Richtlinie (1992): Richtlinie 92/43/EWG des Rates zur Erhaltung der Lebensräume sowie der wildlebenden Tiere und Pflanzen vom 21. Mai 1992, Abl. Nr. L 206, S. 7.
Ficetola, G. F., Padoa-Schioppa, E., Monti, A., Massa, R., De Bernardi, F., Bottoni, L. (2004): The importance of aquatic and terrestrial habitat for the European pond turtle (Emys orbicularis): implications for conservation planning and management. Can. J. Zool. 82, 1704-1712.

Funk, A., Reckendorfer, W., Kucera-Hirzinger, V., Raab, R., Schiemer, F. (2009): Aquatic diversity in a former floodplain: remediation in an urban context. Ecol. Eng. 35, 1476-1484.

Funk, A., Gschöpf, C., Blaschke, A. P., Weigelhofer, G., Reckendorfer, W. (2013): Ecological niche models for the evaluation of management options in an urban floodplain-conservation 
vs. restoration purposes. Environmental Science \& Policy, 34, 79-91.

Frühauf, J., Donnerbaum, K., Raab, R., Wichmann, G. (2006): Habitatnutzung des Eisvogels im Bereich Orth an der Donau. Beweissicherung Eisvogel - Orth. Studie im Auftrag der Nationalpark Donau-Auen GmbH im Rahmen des LIFE-Projektes Gewässervernetzung und Lebensraummanagement Donauauen

Gerds, T. A., Cai, T., Schumacher, M. (2008): The performance of risk prediction models. Biometrical J. 50, 457-479.

Guti, G. (1996): Species composition of juvenile (0+) fish assemblages in the Szigetköz floodplain of the Danube. Tiscia 30, 49-54.

Hanley, J. A., McNeil, B. J. (1982): The meaning and use of the area under a receiver operating characteristic curve. Radiology 143, 29-36.

Hein, T., Heiler, G., Pennetzdorfern, D., Riedler, P., Schagerl, M., Schiemer, F. (1999): The danube restoration project: functional aspects and planktonic productivity in the floodplain system. Reg. Riv. Res. Manag., 15: 259-270.

Hein, T., Blaschke, A. P., Haidvogl, G., Hohensinner, S., Kucera-Hirzinger, V., Muhar, S., Preiner, S., Reiter, K., Schuh, B., Weigelhofer, G., Zsuffa, I. (2006): Optimised management strategies for the Biosphere reserve Lobau, Austria based on a multi criteria decision support system: using ecohydrological model approaches. Ecohydrology and Hydrobiology, 6, 25-36.

Hein, T., Schwarz, U., Habersack, H., Nichersu, I., Preiner, S., Willby, N., Weigelhofer, G. (2015) Current status and restoration options for floodplains along the Danube River Science of the total Environment, 10.1016/j.scitotenv.2015.09.07 Hohensinner, S., Lager, B., Sonnlechner, C. Haidvogl, G., Gierlinger, S., Schmid, M., Winiwarter, V. (2013): Changes in water and land: the reconstructed Viennese riverscape from 1500 to the present. Water History, 5 (2), 145-172; ISSN 1877-7236

Kolozsvary, M. B., Swihart, R. K. (1999): Habitat fragmentation and the distribution of amphibians: patch and landscape correlates in farmland. Can. J. Zool. 77, 1288-1299.
Maletzky, A., Kyek, M., Goldschmid, A. (2007): Monitoring status, habitat features and amphibian species richness of Crested newt (Triturus cristatus superspecies) ponds at the edge of the species range (Salzburg, Austria), Ann. Limnol. Int. J. Lim. 43, 107-115.

Malmqvist, B., Rundle, S. (2002): Threats to the running water ecosystems of the world. Environmental conservation, 29(02), 134-153.

NÖ NschG - NÖ Artenschutzverordnung (2005): VO LRNI/5500/02 LGBI. 5500-3 vom 12. August 2005

Oldham, R. S., Keeble, J., Swan, M. J. S, Jeffcote, M. (2000): Evaluating the suitability of habitat for the great crested newt (Triturus cristatus). Herpetological Journal 10, 143-155.

Reckendorfer, W., Schmalfuss, R., Baumgartner, C., Habersack, H., Hohensinner, S. et al (2005): The Integrated River Engineering Project for the free-flowing Danube in the Austrian Alluvial Zone National Park: contradictory goals and mutual solutions. Archiv für Hydrobiologie, Supplement Large Rivers 155, 613-630.

Reckendorfer, W., Funk, A., Gschöpf, C., Hein, T., Schiemer, F. (2013): Aquatic ecosystem functions of an isolated floodplain and their implications for flood retention and management. Journal of Applied Ecology 50 (1), 119-128.

Sommerwerk, N., Bloesch, J., Paunovic, M. Baumgartner, C., Ostojic, A., Siber, R., Schneider-Jacoby, M., Hein, T., Tockner, K. (2009): The Danube River Basin. In: K. Tockner, U. Uehlinger and C. T. Robinson (eds), Rivers of Europe, Academic Press, 59-112.

Sommerwerk, N., Bloesch, J., Paunovic, M. Baumgartner, C., Venohr, M., Schneider-Jacoby, M., Hein, T., Tockner, K. (2010): Managing the world's most international river: the Danube River Basin. Marine and Freshwater Research 61, 736-748.

Schiemer, F., Spindler, T. (1989): Endangered fish species of the Danube river in Austria. Regul. Rivers: Res. Manage. 4: 397-407.

Schiemer, F., Waidbacher, H. (1992): Strategie for conservation of a Danubian fish fauna. River Conservation and Management. P. J. Boon, P. Ca- low and G. E. Petts (editor). Verlag John Wiley \& Sons Ltd.: 363-382.

Schiemer, F., Baumgartner, C., Tockner, K (1999): Restoration of floodplain rivers: The Danube Restoration Project. Reg. Riv. Res. Manag., 15: 231-244.

Schiemer, F, Hein, T., Reckendorfer, W. (2007): Key-concept for large river restoration. Ecohydrology and Hydrobiology 7 (2), 101-111

Schwarz, U. (2010): Assessment of the restoration potential along the Danube and main tributaries. For WWF International Danube-Carpathian Programme, Vienna, 58p.

Tockner, K., Pennetzdorfer, D., Reiner, N. Schiemer, F., Ward, J. V. (1999): Hydrological connectivity, and the exchange of organic matter and nutrients in a dynamic river-flood-plain system (Danube, Austria). Freshwat. Biol., 41: 521-535.

Tockner, K., Stanford, J. A. (2002): Riverine flood plains: present state and future trends. Environmental conservation, 29(03), 308-330.

Vogelschutz-Richtlinie (2009): Richtlinie 2009/ 147/EG des europäischen Parlaments und des Rates über die Erhaltung der wildlebenden Vogelarten vom 30. November 2009, Abl. Nr. L 20 S. 7

Weigelhofer, G., Hein, T., Kucera-Hirzinger, V., Zornig, H., Schiemer, F. (2011): Hydrological improvement of a former floodplain in an urban area: potential and limits. Ecol. Eng. 37, 1507-1514.

Weigelhofer, G., Reckendorfer, W., Funk, A. Hein, T. (2013): Auenrevitalisierung-Potenzial und Grenzen am Beispiel der Lobau, Nationalpark Donau-Auen. Österreichische Wasser-und Abfallwirtschaft, 65(11-12), 400-407.

Wr. NschG - Wr. Naturschutzverordnung (1998) VO RWI/L480/020. LGBl für Wien Nr. 45/1998 Wintle, B., Elith, J., Potts, J. M. (2005): Fauna habitat modelling and mapping: a review and case study in the Lower Hunter Central Coast region of NSW. Austral. Ecol. 30, 719-738. 